EPJ Web of Conferences 20, 03004 (2012)

DOI: $10.1051 /$ epjconf/20122003004

(C) Owned by the authors, published by EDP Sciences, 2012

\title{
Towards Dense Nuclear Matter in A Modified Sakai-Sugimoto Model
}

\author{
Youngman Kim* ${ }^{* \dagger}$, Mannque Rho ${ }^{\ddagger}$, Takuya Tsukioka* and Deokhyun $\mathrm{Yi}^{*}$ \\ * Asia Pacific Center for Theoretical Physics, Pohang, Gyeongbuk 790-784, Korea \\ $\dagger$ Department of Physics, Pohang University of Science and Technology, Pohang, \\ Gyeongbuk 790-784, Korea \\ \$ Institut de Physique Théorique, CEA Saclay, 91191 GIf-sur-Yvette Cédex, France, \\ \& Department of Physics, Hanyang University, Seoul 133-791, Korea
}

\begin{abstract}
As a part of the attempt to address dense baryonic matter, we first review holographic approaches to QCD. The big advantage of the holographic approaches is that they render strongly coupled 4D gauge theories as duals of certain weakly coupled string/supergravity that are well understood. Its relevance to real QCD is one of the central problems in hadron/nuclear physics as well as in the context of applied string theory. None of the models based on these holographic approaches presently available can adequately describe the system we are interested in, namely dense baryonic matter. Nevertheless, some aspects of the holographic approach are found to describe certain processes both in vacuum and in medium. In this talk we only present the structure of a model that appears to be closest to QCD, and has the potential to address the problem.
\end{abstract}

\section{Introduction}

Conventional quantum field theory is mainly based on perturbative techniques and therefore it is hard to address various aspects of strong coupling dynamics such as QCD at low energy. The perturbative methods i.e. small coupling expansion are applicable only in the energy regime much higher than $\Lambda_{\mathrm{QCD}}$ on which the QCD coupling becomes order one. (See for example [1] to get some aspects of the dense quark matter in the perturbation regime.) Most distinguishable features of QCD are confinement/deconfinement and chiral symmetry breaking/restoration. The dynamical origin of these experimentally observed aspects is not well-understood so far.

Lattice QCD is one of the powerful nonperturbative techniques and has proven to be invaluable in guiding models to describe hadronic matter at high temperature although there are some limitations to describe non-equilibrium time dependent dynamics. (See for example [2] for recent review.) However due to the famous sign problem, lattice QCD cannot access, at least at the moment, the high density regime which is physically interesting, e.g., compact stars

In recent years, RHIC experiments have probed the strong coupling regime of QCD at high temperature, exposing an ideal fluid nature of QGP [3] and suggesting the ratio of viscosity to entropy density to be close to the bound $\eta / s=1 /(4 \pi)$. This has been calculated by using AdS/CFT correspondence in string theory [4]. The dual gravity theory that yields this result is not QCD, so what figures in this phenomenon must be universality in action, not specific dynamics. Indeed this appears to be the case for any gauge theories within the Einstein gravity dual in the framework of AdS/CFT correspondence.

Whether or not this kind of universality holds in hadronic matter at high density is totally unknown. In this talk, we make the first step in addressing dense matter in the context of a holographic QCD approach based on the AdS/CFT correspondence. As stated, for dense matter, lattice QCD does not help much, and furthermore, no model-independent QCD-motivated theoretical approaches are

This is an Open Access article distributed under the terms of the Creative Commons Attribution-Noncommercial License 3.0, which permits unrestricted use, distribution, and reproduction in any noncommercial medium, provided the original work is properly cited. 


\section{EPJ Web of Conferences}

available. On the other hand, recent studies using the holographic QCD approaches show the potential to unravel what happens in dense matter but there are no realistic holographic QCD models at the moment. In addressing this problem, what we do here is merely to clarify what ingredients are needed in the Sakai-Sugimoto model presently considered to be the only holographic QCD model that has the correct structure for the problem, namely, chiral symmetry and confinement and found to be successful for certain processes dominated by large $N_{\mathrm{c}}$. We discuss a modified D4/D8 model with tachyons. We will point out at the end what ingredients may be indispensable in the model in light of what is known in QCD-inspired models. No results will be reported in this talk, relegating them to a future publication.

\section{AdS/CFT correspondence}

The AdS/CFT correspondence is a (gravity theory)/(gauge theory) duality in string theory. This correspondence is based on two ideas. One is holography which relates theories living in spaces with different dimensionality. In high energy physics, the holography is introduced as the statement: quantum gravity in some region may be described in terms of a non gravitational theory living in its boundary [5]. Another important idea is that the nonabelian gauge theory might have a dual description in terms of strings [6]. These two can be realized with precise meaning through the AdS/CFT correspondence [7].

\subsection{Basics in string theory}

We start by giving some basic aspects about string theory. One dimensional object called string is a fundamental player. There are two types of strings; open strings which are the line segments with two end points and closed strings which are loops. One of the fundamental parameters of the theory is the string length $l_{\mathrm{s}}=\sqrt{\alpha^{\prime}}$, which is built in the string tension $T_{\mathrm{s}}=1 /\left(2 \pi \alpha^{\prime}\right)$. The strength of interaction between strings given by the dimensionless string coupling $g_{\mathrm{s}}$ is another one. Open strings originally showed up as QCD strings and succeeded to explain some aspects in hadron physics such as Venetiano amplitude and Regge trajectory. Imposing some consistencies, it turns out that the target spacetime where strings can live is not 4D but 10D for superstring. We here consider this critical superstring theory. However, once it was shown that the closed string contains spin 2 massless mode which can be regarded as graviton, string theory was extensively developed from the point of view of the unified picture of the elementary forces. This unified framework makes it possible to discuss the gauge/gravity correspondence. In order to describe 4D physics, the compactification procedure is essential and it has been shown that this internal space was big enough to provide the gauge group of the standard model.

In string theories, not only strings but also certain kind of extended objects called $\mathrm{D} p$-branes in analogy with membrane can be consistently formulated [8]. The $\mathrm{D}$ (irichlet) $p$-brane is a $p$-dimensional extended solitonic object in 10D spacetime and gives the place where open strings can end with Dirichlet boundary conditions on the transverse directions to the brane. These open string excitations make D-brane fluctuate. End points of open string have charges and therefore open string can describe the dynamics of the gauge field. In the world volume of D-brane, gauge fields can live, so that we could discuss 4D gauge theories through 4D world volume of D3-brane, for example. One could obtain nonabelian gauge dynamics by introducing some numbers of D-branes [9]. Open strings ending on the same D-brane yield massless vector multiplets, while those ending on the different D-branes give rise to those for massive vector. Since in this situation, the string which has a tension $1 /\left(2 \pi \alpha^{\prime}\right)$ turns out to have a nonzero length, this stretched string acquires the mass $m=$ (distance) $/\left(2 \pi \alpha^{\prime}\right)$. If the $N$ D-branes coincide, the masses of these modes vanish and then we obtain an enhanced $\mathrm{U}(N)$ symmetry with $N^{2}$ vector fields. The overall U(1) factor corresponds to the overall position of the branes and may decouple from the $\mathrm{SU}(N)$ gauge dynamics.

On the other hand, if $\mathrm{D} p$-branes exist in the 10D bulk spacetime, the spacetime will be curved through the backreaction, especially for the case where large numbers of branes are considered. In 
fact, it was shown that the existence of $\mathrm{D} p$-branes can be realized as the solution of supergravity which is the low energy effective theory in superstring theory (type IIA, IIB closed string) [10]. To be more specific, we consider $N$ coincident D3-branes, whose supergravity solution for the metric is given by

$$
(\mathrm{d} s)^{2}=\left(1+\frac{L^{4}}{r^{4}}\right)^{-1 / 2}\left(-(\mathrm{d} t)^{2}+(\mathrm{d} \mathbf{x})^{2}\right)+\left(1+\frac{L^{4}}{r^{4}}\right)^{1 / 2}\left((\mathrm{~d} r)^{2}+r^{2} \mathrm{~d} \Omega_{5}^{2}\right), \quad \text { with } \quad L^{4}=N\left(4 \pi g_{\mathrm{s}} l_{\mathrm{s}}^{4}\right),
$$

where $(t, \mathbf{x})$ and $\left(r, \Omega_{5}\right)$ stand for the coordinates for D3-brane (4D) and for their transverse 6D spacetime which is decomposed by its radial and $\mathrm{S}^{5}$ parts, respectively. The D3-branes sit at $r=0$.

\subsection{Field theory (or decoupling) limit and $\mathrm{AdS}_{5} \times \mathrm{S}^{5}$}

We have two descriptions of the $N$ D3-brane in 10D bulk spacetime. One is from open string point of view which allows one to consider the gauge theory which is $\mathcal{N}=4 \mathrm{SU}(N)$ super Yang-Mills in 4D in this case. The other point of view comes from the closed string description treated in supergravity approximation. Since the underlying configuration is the same, by looking into the detail of the two descriptions and taking certain limits, we can obtain new insights from them. This is the AdS/CFT correspondence.

In the open string description, there are open strings ending on these D3-branes and also closed strings in the bulk. Through the low energy effective action of D3-branes given by the Dirac-BornInfeld action [11], Yang-Mills gauge coupling can be related with the parameter in the string theory $g_{\mathrm{YM}}=2 \pi g_{\mathrm{s}}$. If we are interested in the gauge theory itself, we need to take the decoupling limit where bulk gravitation modes coming from closed string may be neglected. Since 10D gravitational coupling is given by $G_{10}=(2 \pi)^{6} l_{\mathrm{s}}^{8} g_{\mathrm{s}}^{2} / 8$, this is achieved by taking the limit; $g_{\mathrm{YM}}$ finite and $G_{10} \rightarrow 0$, i.e. $\alpha^{\prime} \rightarrow 0$. As a result, the bulk supergravity and $\mathcal{N}=4 \mathrm{SU}(N)$ super Yang-Mills in 4D are totally decoupled.

In the closed string (supergravity) description, we also consider low energy excitations of the system. First of all, the geometry given in (1) is divided into three parts: the asymptotic region $r \rightarrow \infty$ which is flat, the near horizon region $r \rightarrow 0$ and the intermediate region called the "throat" region. Due to the red-shift factor in the metric (1), an observer in the asymptotic region will measure an energy $E_{\infty}$ which is smaller than the original energy $E_{r}$,

$$
E_{\infty}=\sqrt{g_{00}} E_{r}=\left(1+\frac{L^{4}}{r^{4}}\right)^{-1 / 4} E_{r} .
$$

Even for a finite energy $E_{r \sim 0}$, the measurement $E_{\infty}$ tends to 0 . Closed strings which provide these excitations in the throat region are trapped there. The throat region looks like a gravitational potential well, in which there exist several kinds of normalizable modes observed in the dual gauge theory. The excitations observed weakly in the asymptotic region could fall into two types: low energy excitations in the bulk and finite energy excitations near the horizon. These are decoupled and each excitations follow supergravity theories in the bulk and in the near horizon, respectively. In the near horizon $r \rightarrow 0$ $\left(u=r / \alpha^{\prime}:\right.$ fix $\left.\left(\alpha^{\prime} \rightarrow 0\right)\right)$, the metric (1) reduces to

$$
(\mathrm{d} s)^{2}=\frac{r^{2}}{L^{2}}\left(-(\mathrm{d} t)^{2}+(\mathrm{d} \mathbf{x})^{2}\right)+\frac{L^{2}}{r^{2}}(\mathrm{~d} r)^{2}+L^{2} \mathrm{~d} \Omega_{5}^{2},
$$

which is $\mathrm{AdS}_{5} \times \mathrm{S}^{5}$. The parameter $L$ corresponds to the radius of the curvature of $\mathrm{AdS}_{5}$ and $\mathrm{S}^{5}$.

Comparing these two descriptions in low energy, Maldacena conjectured that $\mathcal{N}=4$ super YangMills in 4D is equivalent to the supergravity or (small $\alpha^{\prime}$ limit of) type IIB string theory on $\mathrm{AdS}_{5} \times \mathrm{S}^{5}$. $\mathrm{AdS}_{5}$ spacetime consists of $4 \mathrm{D}$ part $(t, \mathbf{x})$ whose metric is $4 \mathrm{D}$ flat spacetime times conformal factor $r^{2} / L^{2}$ and an extra "holographic" direction $r$.

\subsection{Correspondence}

Let us discuss the above conjecture more precisely. 


\section{EPJ Web of Conferences}

We first compare symmetries in the theories. It should be mentioned that the global symmetries in the gauge theory are dual to the gauge symmetry in the gravity theory. The geometry $\operatorname{AdS}_{5} \times S^{5}$ has the isometries $\mathrm{SO}(4,2)$ and $\mathrm{SO}(6)$. These symmetries correspond to the global symmetries for $4 \mathrm{D}$ conformal group and R-symmetry $S U(4) \simeq S O(6)$ in $\mathcal{N}=4$ super Yang-Mills theory.

The conformal group $\mathrm{SO}(4,2)$ contains the subgroup $\mathrm{SO}(1,1)$ which is realized as the scaling symmetry of the metric (2): $(t, \mathbf{x}, r) \rightarrow\left(\Lambda t, \Lambda \mathbf{x}, \Lambda^{-1} r\right)$ with a constant $\Lambda$. This immediately implies that the radial (alias holographic) coordinate $r$ can be understood as the energy scale for the boundary 4D theory [12]. In fact, the renormalization group equation in the field theory can be computed within the framework of supergravity in AdS spacetime [13]. The radial coordinate plays a role of the renormalization scale. Therefore, in general, the supersymmetric gauge theory at the UV fixed point is living in the boundary of the AdS spacetime. Its RG flow to the IR corresponds to going into the throat region.

We next consider the parameters of the two theories. In Yang-Mills theory, there are two parameters i.e. the gauge coupling $g_{\mathrm{YM}}$ and $N$ for the gauge group $\mathrm{SU}(N)$. In the gravity or string side, we have $\alpha^{\prime}, L$ and string coupling $g_{\mathrm{s}}$. These are related by

$$
g_{\mathrm{YM}}^{2}=2 \pi g_{\mathrm{s}} \quad \text { and } \quad N=\frac{L^{4}}{4 \pi g_{\mathrm{s}} \alpha^{\prime 2}} \text {. }
$$

We introduce 't Hooft coupling $\lambda \equiv N g_{\mathrm{YM}}^{2}$ which is a relevant loop expansion parameter rather than $g_{\text {YM }}$ in large $N$ gauge theory. Then we rewrite the relation (3) as

$$
\lambda=\frac{L^{4}}{2 \alpha^{\prime 2}}, \quad \text { and } \quad \frac{N}{\lambda}=\frac{1}{2 \pi g_{\mathrm{s}}} .
$$

The first relation in (4) implies that strong coupling region in Yang-Mills theory can be analyzed by the supergravity which is applicable for a weakly curved spacetime i.e. $L^{2} \geq \alpha^{\prime}$. At fixed $\lambda$, the second relation states that $1 / N^{2}$ corresponds to the string loop expansion parameter $g_{\mathrm{s}}^{2}$. Therefore, large $N$ Yang-Mills corresponds to tree level string theory and classical supergravity for large $\lambda$.

Quantitative correspondence can be given by the path integral formulation of the two theories [14]. These partition functions should be equated in the AdS/CFT correspondence.

$$
Z_{\mathrm{CFT}} \equiv Z_{\text {string }} \text {. }
$$

It is known that there exist maps between the fields $\Phi(x, r)$ in the bulk gravity side and the local gauge invariant operators $O(x)$ in the 4D gauge theory side. For simplicity, compactifing $\mathrm{S}^{5}$ part, we here only consider $5 \mathrm{D} \mathrm{AdS} 5$ bulk spacetime. It may be convenient to rewrite the metric by introducing a dimensionless coordinate $\rho \equiv L / r$. Then the $\mathrm{AdS}_{5}$ part of the metric (2) becomes

$$
(\mathrm{d} s)^{2}=\frac{1}{\rho^{2}}\left(-(\mathrm{d} t)^{2}+(\mathrm{d} \mathbf{x})^{2}+L^{2}(\mathrm{~d} \rho)^{2}\right) .
$$

The boundary is now located at $\rho=0$

Considering a fluctuation of the "background" in the $N$ D3-brane system, the following coupling may be introduced in the original action in the boundary gauge theory side,

$$
S_{0}[\varphi] \longrightarrow S_{0}[\varphi]+\int \frac{\mathrm{d}^{4} x}{\partial(\operatorname{AdS})}(x) O(x)
$$

where $\varphi$ stands for the field on the D-branes. $J(x)$ corresponds to the fluctuation of the background field on the string theory and can be regarded as the source term for the gauge field operator $O(x)^{1}$.

${ }^{1}$ For example, one can obtain the following couplings

$$
S_{0}[\varphi]+\int \mathrm{d}_{\partial(\mathrm{AdS})}{ }^{4} x\left(h_{\mu v} T^{\mu v}+\phi \operatorname{Tr} F_{\mu v}^{2}\right)
$$

where the sources $h_{\mu v}(x)$ and $\phi(x)$ are fluctuations of the metric and the dilaton, respectively. Here $T_{\mu \nu}(x)$ is the energy-momentum tensor of the gauge theory and $F_{\mu \nu}(x)$ is the field strength. 
From the bulk gravity theory point of view, this coupling originates from the asymptotic excitation of the bulk field. One can think of the source $J(x)$ as the boundary value of the bulk field $\Phi(x, \rho)$. In the bulk, there are two kinds of fluctuations which are two independent solutions of the classical equation of motion for $\Phi(x, \rho)^{2}$. Near the boundary, we assume that the solution can be written as the Frobenius solution with "integration constants" $\mathcal{A}(x)$ and $\mathcal{B}(x)$ as

$$
\Phi_{\mathrm{cl}}(x, \rho)=\mathcal{A}(x) F^{(I)}(\rho)+\mathcal{B}(x) F^{(I I)}(\rho),
$$

with

$$
\begin{aligned}
& F^{(I)}(\rho)=\rho^{n}(1+O(\rho))+c(x) F^{(I I)}(\rho) \log \rho, \\
& F^{(I I)}(\rho)=\rho^{m}(1+O(\rho))
\end{aligned}
$$

where the exponents $m>n$ at the boundary depend on the conformal dimensions of the operator $O(x)$ dual to the field $\Phi(x, \rho)^{3}$. Coefficients in the series expansion of the basis $F^{(I)}(\rho)$ and $F^{(I I)}(\rho)$ including $c(x)$ can be obtained recursively except for that of $\rho^{m-n}$ in $F^{(I)}(\rho)$. We may choose this to be zero for convenience. One of the integration constants $\mathcal{A}(x)$ can be fixed at the boundary and may be identified with the source $J(x)$. The remaining constant could be fixed in the bulk imposing "regularity" condition, so that the solution $\Phi_{\mathrm{cl}}(x, \rho)$ can be completely fixed. In general, the mode for $F^{(I)}(\rho)$ scales in a non-normalizable way with $\rho$, while that for $F^{(I I)}(\rho)$ is normalizable [15].

In the classical supergravity approximation, where the saddle point approximation is available, the AdS/CFT correspondence (5) is thus formulated as

$$
\int \mathcal{D} \varphi \mathrm{e}^{i S_{0}[\varphi]+i \int \mathrm{d}^{4} x J(x) O(x)}=Z_{\mathrm{CFT}}[J]=Z_{\mathrm{CFT}} \equiv Z_{\text {string }} \approx Z_{\mathrm{SUGRA}}\left[\Phi_{\mathrm{cl}}\right]=\mathrm{e}^{i S_{\text {SUGRA }}\left[\Phi_{\mathrm{cl}}\right]}
$$

with

$$
J(x)=\mathcal{A}(x),
$$

where $S_{\text {SUGRA }}\left[\Phi_{\mathrm{cl}}\right]$ is an on-shell action with the solution $\Phi_{\mathrm{cl}}(x, \rho)$. Since the left hand side of the equation (6) can be regarded as the generating functional with the source $J(x)$, taking simply the functional variation of the on-shell action $S_{\text {SUGRA }}\left[\Phi_{\mathrm{cl}}\right]$ with respect to $\mathcal{A}(x)(=J(x))$, one can obtain correlation functions of $O(x)$ in the gauge field side. In particular, the coefficient of the normalizable mode $\mathcal{B}(x)$ would be proportional to the VEV of the operator $O(x)$.

The correlation functions in general suffer from divergences at the boundary. Analogous to the renormalization in the quantum field theories, those could be cured by adding appropriate local counter terms in the boundary without spoiling the equation of motion in the bulk. $S_{\text {SUGRA }}\left[\Phi_{\mathrm{cl}}\right]$ in (6) should be understood as the renormalized on-shell action [16].

After the AdS/CFT correspondence, many modifications of the original conjecture have been proposed and studied. One of the directions is to modify the gravity side in a suitable manner in order to observe some features in the gauge theory side.

\section{Sakai-Sugimoto Model}

One of the major goals in the gauge/gravity dualities is to find holographic models which capture features of QCD, in other word, to find the dual "geometry" for QCD. A model which includes the confinement and the chiral symmetry breaking can be obtained from the decoupling limit of a D4brane background with D8-branes added to introduce flavors. This is the Sakai-Sugimoto model [17].

\footnotetext{
${ }^{2}$ One can introduce the Fourier transformation for the 4D part i.e. $x^{\mu} \leftrightarrow k^{\mu}$. The equation of motion to be considered here is the second order ordinary differential equation for the holographic direction $\rho$.

${ }^{3}$ If $m-n$ is not an integer, the term proportional to $\log \rho$ may be absent.
} 


\section{EPJ Web of Conferences}

\subsection{D4-brane background}

Witten proposed a construction of the holographic dual of 4D pure $\mathrm{SU}\left(N_{\mathrm{c}}\right)$ Yang-Mills theory [18]. One starts with $N_{\mathrm{c}}$ D4-branes on which 5D supersymmetric $\mathrm{SU}\left(N_{\mathrm{c}}\right)$ gauge is defined and further compactifies one spatial circle with radius $1 / M_{\mathrm{KK}}$. One imposes the anti-periodic boundary conditions for the world volume fermions on this circle, so that the supersymmetry is explicitly broken. Not only fermions are massive in tree-level, but also scalar fields on the branes acquire masses through oneloop effect. After imposing the field theory (or decoupling) limit which is large $N_{\mathrm{c}}$ limit in this case, at the low energies compared to the string scale, the theory is 5D pure Yang-Mills with a dimensionful 't Hooft coupling $\lambda_{5}=N_{\mathrm{c}}(2 \pi)^{2} l_{\mathrm{s}} g_{\mathrm{s}}$. At the energies in which Kaluza-Klein modes are suppressed, the theory is supposed to reduce to pure Yang-Mills in $4 \mathrm{D}^{4}$. As we discussed in the case of D3-branes, we can consider the gravity dual description. Indeed, the system has a dual geometry which is given by the Type IIA supergravity solution in 10D:

$$
\begin{aligned}
(\mathrm{d} s)^{2} & =\left(\frac{U}{R}\right)^{3 / 2}\left\{-(\mathrm{d} t)^{2}+(\mathrm{d} \mathbf{x})^{2}+f(U)\left(\mathrm{d} x^{4}\right)^{2}\right\}+\left(\frac{R}{U}\right)^{3 / 2}\left\{\frac{(\mathrm{d} U)^{2}}{f(U)}+U^{2}\left(\mathrm{~d} \Omega_{4}\right)^{2}\right\}, \\
\mathrm{e}^{\phi} & =g_{s}\left(\frac{U}{R}\right)^{3 / 4}, \quad F_{4}=\mathrm{d} C_{3}=\frac{2 \pi N_{c}}{V_{4}} \epsilon_{4},
\end{aligned}
$$

with

$$
f(U)=1-\left(\frac{U_{\mathrm{KK}}}{U}\right)^{3}, \quad R^{3}=\pi g_{s} N_{c} l_{s}^{3} .
$$

Here $\phi$ is the dilaton and $F_{4}$ is the four-form field strength of Ramond-Ramond field. The coordinates $\left(t, \mathbf{x}, x^{4}\right)$ parameterize 5D world volume of D4-brane, where $x^{4}$ is compactified on $\mathrm{S}^{1} . U$ is the radial coordinate which is transverse to the D4-brane. $\left(\mathrm{d} \Omega_{4}\right)^{2}$ and $\epsilon_{4}$ are line element and volume form on $\mathrm{S}^{4}$, respectively, and $V_{4}=8 \pi^{2} / 3$ is its volume. Since the period of $x^{4}$ is $2 \pi / M_{\mathrm{KK}}$, a conical singularity at $U=U_{\mathrm{KK}}$ in the radial coordinate may be removed by imposing the relation

$$
M_{\mathrm{KK}}^{2}=\frac{9 U_{\mathrm{KK}}}{4 R^{3}},
$$

which makes the $\left(U, x^{4}\right)$ submanifold a cigar-like form with a tip at $U=U_{\mathrm{KK}}$ where the $x^{4}$-circle shrinks to zero.

We should mention the regime of validity of supergravity approximation. We impose that the spacetime curvature be much smaller than the string length scale. The maximum value of the Ricci scalar of the background goes to $\left(R^{3} U_{\mathrm{KK}}\right)^{-1 / 2}$, so that the condition $\left(R^{3} U_{\mathrm{KK}}\right)^{1 / 4} \gg l_{\mathrm{s}}$ gives

$$
\lambda_{4}=\lambda_{5} \frac{M_{\mathrm{KK}}}{2 \pi} \gg 1,
$$

which implies the large 't Hooft coupling limit.

Since the dilaton field is not constant in this background, string loop effects cannot be suppressed for large $U$. The critical value $U$ where the dilaton is order one can be estimated as $U_{\text {cutoff }} \sim N_{\mathrm{c}}^{4 / 3} l_{\mathrm{s}}^{2} / \lambda_{5}$. Beyond this one has to lift the background to more upper description in 11D M-theory [19].

\subsection{Finite temperature}

In order to study thermal gauge theories, we may take the imaginary time analysis ${ }^{5}$. We analytically continue to Euclidean spacetime and compactify the time direction with the thermal period $1 / T$ with

\footnotetext{
${ }^{4}$ In [18], it has been shown that the theory develops a mass gap. In the regime $\lambda_{5} \ll 1 / M_{\mathrm{KK}}$, the scale of the mass gap is exponentially suppressed compared to $M_{\mathrm{KK}}$. Then the gauge theory is effectively 4D pure Yang-Mills. However in the strong coupling regime $\lambda_{5} \gg 1 / M_{\mathrm{KK}}$, where the dual gravity approximation is available (see (8)), the gauge theory develops the mass gap of the order $1 / M_{\mathrm{KK}}$ and therefore Kaluza-Klein modes do not decouple.

5 For non-equilibrium systems, we can use the real time analysis given by the Schwinger-Keldysh formalism, instead. In the framework of the AdS/CFT correspondence, there is a prescription to calculate Green functions in the real time [20].
} 
temperature $T$. In the gravity side, one can simply use the Euclidean version of the metric (7a) with the thermal circle for $t$. There is another nontrivial metric with the same asymptotics. This is given by exchanging the coordinates $t$ and $x^{4}$ in the Euclidean version of the metric (7a):

$$
(\mathrm{d} s)^{2}=\left(\frac{U}{R}\right)^{3 / 2}\left\{f(U)(\mathrm{d} t)^{2}+(\mathrm{d} \mathbf{x})^{2}+\left(\mathrm{d} x^{4}\right)^{2}\right\}+\left(\frac{R}{U}\right)^{3 / 2}\left\{\frac{(\mathrm{d} U)^{2}}{f(U)}+U^{2}\left(\mathrm{~d} \Omega_{4}\right)^{2}\right\},
$$

where $f(U)=1-\left(U_{\mathrm{T}} / U\right)^{3}$ with a new constant $U_{\mathrm{T}}$. The dilaton and the Ramon-Ramond field strength are the same as (7b). This Euclidean black hole, which is a thermal object, gives the Hawking temperature $T$,

$$
T^{2}=\frac{9 U_{\mathrm{T}}}{16 \pi^{2} R^{3}} .
$$

This is obtained by removing the conical singularity for the thermal circle $t$ at the horizon $U=U_{\mathrm{T}}$ with the period $1 / T$, as we did for the circle $x^{4}$ in (7a). Therefore at finite temperature, there are two gravity backgrounds in which each of them has two circles. Nature chooses one of them in the economical way. Comparing the free energies defined by the thermal partition function with the source i.e. $F=-T \log Z[J]=T S_{\text {SUGRA }}^{\mathrm{E}}\left[\Phi_{\mathrm{cl}}\right]$ where the Euclidean version of the correspondence (6) is used, it has been shown that there is a first order phase transition between two of them at $T=M_{\mathrm{KK}} /(2 \pi)$, where these two become essentially the same due to $U_{\mathrm{KK}}=U_{\mathrm{T}}$. At lower temperatures $T<M_{\mathrm{KK}} /(2 \pi)$, the Euclidean version of (7a) is energetically favored, while at higher temperatures $T>M_{\mathrm{KK}} /(2 \pi)$ the background (9) is favored $[18,21]$. This can be understood intuitively by comparing the two periods of circles i.e. $1 / T$ for $t$ and $2 \pi / M_{\mathrm{KK}}$ for $x^{4}$. The metric having the smaller circle which can shrink to zero is always chosen. The computation for the free energies of the two phases shows that their scales are $N_{\mathrm{c}}^{0}$ and $N_{\mathrm{c}}^{2}$ for the low temperature and the high temperature phases, respectively. This means, in the low (high) temperature phase, the gauge degrees of freedom are confined (deconfined) [18,21].

\subsection{Adding flavors}

The D-branes considered in the previous section are "color" branes. Two endpoints of open strings which correspond to point charges in the fundamental/anti-fundamental representations are attached to the same branes, so that they transform in the adjoint representation of the $\mathrm{SU}\left(N_{\mathrm{c}}\right)$ gauge group. In order to consider the fundamental matter with flavors, we need to introduce open string whose only one endpoint is attached to the color branes. This can be realized by introducing different types of D-branes. Since the fundamental matter lives in $4 \mathrm{D}$, this $4 \mathrm{D}$ spacetime should be filled by these $\mathrm{D} p$-branes $(p \geq 3)$.

In order to accommodate these "flavor" branes in the dual gravity description, we need to find a solution of supergravity in the presence of the flavor branes together with the color branes. Since these branes will communicate with each other, it is in general not easy to pursue this direction. What we can do here is to neglect the backreaction of the flavor branes. This can be realized if one adds much smaller number of flavor branes $N_{\mathrm{f}}$ compared to that of color branes i.e. $N_{\mathrm{f}} \ll N_{\mathrm{c}}$ [22]. This is the probe brane approximation which is similar to the quenched approximation in lattice QCD in studying the effect of a test quark in the given gluon background. It is easy to see that the Newton potential produced by the flavor branes is suppressed by $N_{\mathrm{f}} / N_{\mathrm{c}}$. In this probe limit, the color branes simply yield the background and in this background the flavor branes are embedded so as to extremize their action. In the gauge theory side, in the decoupling limit, the gauge symmetry of the added flavor branes becomes a global symmetry, since the gauge coupling for the flavor branes vanishes.

We here take D8 and $\overline{\mathrm{D} 8}$ branes as the flavor branes and put $N_{\mathrm{f}}$ pairs of D8 and $\overline{\mathrm{D}} 8$-branes with the following intersection

$$
\begin{array}{rcccc}
t & \mathbf{x} & x^{4} & U & \Omega_{4} \\
N_{\mathrm{c}} \mathrm{D} 4: \times & \times & \times & & \\
N_{\mathrm{f}} \mathrm{D} 8 / \overline{\mathrm{D} 8}: \times & \times & & \times & \times
\end{array}
$$

Here " $x$ " implies that the branes are extended in the corresponding directions, while the blank represents that the branes are point-like in those directions. $N_{\mathrm{f}} \mathrm{D} 8$-branes and $N_{\mathrm{f}} \overline{\mathrm{D} 8}$-branes are placed at 


\section{EPJ Web of Conferences}

the positions $x^{4}=l / 2$ and $x^{4}=-l / 2$ with a separation distance $l$, respectively, These positions are generally functions of the world volume coordinates and determined by the equation of motion of the effective action of these branes.

The low energy effective theory is described by the lightest modes of open strings stretched between (D4, D4), (D4, D8) and (D4, D8). As we discussed, in the decoupling limit, the gauge theories in the D8 and $\overline{\mathrm{D} 8}$-brane world volumes which come from open strings in (D8, D8) and $(\overline{\mathrm{D} 8}, \overline{\mathrm{D} 8})$ are decoupled, so that the symmetries in the gauge theory side are reduced to the global symmetries $\mathrm{U}\left(N_{\mathrm{f}}\right)_{\mathrm{L}}$ and $\mathrm{U}\left(N_{\mathrm{f}}\right)_{\mathrm{R}}$. Open strings in (D4, D4) give pure $\mathrm{SU}\left(N_{\mathrm{c}}\right)$ Yang-Mills as before. Those in (D4, D8) and $(\mathrm{D} 4, \overline{\mathrm{D} 8})$ describe left-handed and right-handed chiral fermions which transform in the fundamental representation of both of $\mathrm{SU}\left(N_{\mathrm{c}}\right)$ color group and $\mathrm{U}\left(N_{\mathrm{f}}\right)_{\mathrm{L}}$ and $\mathrm{U}\left(N_{\mathrm{f}}\right)_{\mathrm{R}}$ flavor group, respectively. Since, in the configuration (10), there are no common transverse directions where strings with finite length can live and give the mass, these fermions are massless. It should be mentioned that open strings in $(\mathrm{D} 8, \overline{\mathrm{D} 8}$ ) could produce the tachyon field. The mass of these string modes might be estimated as

$$
m^{2}=-\frac{1}{2 \alpha^{\prime}}+\left(\frac{\text { distance }}{2 \pi \alpha^{\prime}}\right)^{2}
$$

where the first term is the zero-point energy of the open superstring in the Neveu-Schwarz sector. If the distance between D8 and $\overline{\mathrm{D} 8}$ is large enough, the system could be stable.

In the Sakai-Sugimoto model, we can observe the spontaneous breaking of nonabelian chiral symmetry with new insight. At a large radial position which corresponds to a high energy regime, D8 and $\overline{\mathrm{D} 8}$ branes are well separated and one can see the full $\mathrm{U}\left(N_{\mathrm{f}}\right)_{\mathrm{L}} \times \mathrm{U}\left(N_{\mathrm{f}}\right)_{\mathrm{R}}$ chiral symmetry. However, in the cigar-like background (7a), as the energies are reduced i.e. going into the bulk, D8 and $\overline{\mathrm{D} 8}$ branes have no place to end and consequently join together into the $N_{\mathrm{f}}$ continuous D8 branes at some point in IR while keeping an asymptotic separation $L$ between D8 and $\overline{\mathrm{D} 8}$ branes at large $U$. Therefore D8-branes are embedded in the bulk by forming "U-shape". Here only the diagonal $\mathrm{U}\left(N_{\mathrm{f}}\right)$ symmetry remains unbroken. This is the geometrical realization of the spontaneous chiral symmetry breaking.

Let us consider the finite temperature case where we can discuss the confinement/deconfinement transition. At low temperature, the relevant geometry is given by the Euclidean version of (7a) with the thermal circle for $t$. As we discussed above, the chiral symmetry is broken, since in this regime the D8branes make the U-shape. At high temperature, the corresponding background is (9). This geometry is not the cigar type in the $\left(x^{4}, u\right)$-plane which enforces D8-branes to form U-shape. In this case there are two solutions: the U-shape as before and a "parallel". In the case of the parallel, the D8 and $\overline{\mathrm{D} 8}$ branes end on the black hole horizon separately and consequently there is a full $\mathrm{U}\left(N_{\mathrm{f}}\right)_{\mathrm{L}} \times \mathrm{U}\left(N_{\mathrm{f}}\right)_{\mathrm{R}}$ chiral symmetry. Comparing the free energies of these configurations, it can be shown that in the low temperature regime the U-shaped embedding is the favored one, which implies that the chiral symmetry is broken. At high temperature, the parallel embedding is preferred and therefore the broken symmetry is restored [21].

The baryon chemical potential and density can be also naturally introduced in the model and further make rich structures on the phase diagram in the deconfined phase [23].

\section{Quark mass in a modified Sakai-Sugimoto model}

In spite of many successes to describe QCD qualitatively and in some cases, also quantitatively, there are several open questions in the Sakai-Sugimoto model. One of these is related with the absence of the quark mass and the chiral condensate. (We will mention below what seems to be the most serious one related to the missing scalar meson degree of freedom.) The quark mass matrix in QCD is given in the bi-fundamental representation of $\mathrm{U}\left(N_{\mathrm{f}}\right)_{\mathrm{L}}$ and $\mathrm{U}\left(N_{\mathrm{f}}\right)_{\mathrm{R}}$. In Sakai-Sugimoto model, this bi-fundamental representations comes from the fluctuations of open strings stretched between (D8, $\overline{\mathrm{D} 8}$ ). These modes do not appear in the original model, since these are massive. However, as seen in the equation (11), the mass is controlled by the separation of branes which generally depends on the radial coordinate. Related to this, there is no clear reason to exclude the tachyon field. If the D8 and $\overline{\mathrm{D} 8}$ branes are well 
separated such as UV regime, the original model may be reasonable. However, around the point where these branes join, the tachyonic modes could show up.

In [24], it has been shown that (D8, $\overline{\mathrm{D} 8}$ ) bi-fundamental scalar mode naturally participates into the model in the name of "tachyon" and gives the quark mass and the chiral condensate. Moreover it has been suggested that the tachyon condensate provides the mechanism of chiral symmetry breaking. We would like to close this short review by giving what are addressed in [24]. Alternative approaches to introduce the mass into the model have been considered in [25].

\subsection{Effective action for $D 8 / \overline{D 8}$ with tachyon}

Our D-brane configuration - and their parameterizations - is that sketched in (10) with $N_{\mathrm{f}}=1$ for simplicity. The effective action for $\mathrm{D} p / \overline{\mathrm{D} p}$ branes including the complex tachyon field has been formulated in $[26]^{6}$. Taking the static gauge in the world volume which implies that D8 and $\overline{\mathrm{D} 8}$ branes follow the background geometry, and the unitary gauge for the tachyon field, the effective action in the D4-brane background (7a) and (7b) can be expressed as the sum of those for D8 and $\overline{\mathrm{D} 8}$ branes

$$
S_{\mathrm{D} 8 / \overline{\mathrm{D} 8}}=-\frac{T_{8} V_{4}\left(2 \pi \alpha^{\prime}\right)^{7}}{g_{\mathrm{s}} R^{9}} \int \mathrm{d} t \mathrm{~d}^{3} \mathbf{x} \int \mathrm{d} u V(\mathcal{T}) u^{13 / 4}\left\{\sqrt{D_{\mathrm{L}}[\mathcal{T}(u), h(u)]}+\sqrt{D_{\mathrm{R}}[\mathcal{T}(u), h(u)]}\right\},
$$

where

$$
\begin{aligned}
D[\mathcal{T}(u), h(u)] & \equiv D_{\mathrm{L}}[\mathcal{T}(u), h(u)]=D_{\mathrm{R}}[\mathcal{T}(u), h(u)] \\
& =\frac{u^{3 / 2} f(u)}{4} h^{\prime 2}(u)+\mathcal{T}^{\prime 2}(u)+\mathcal{T}^{2}(u) h^{2}(u)+\frac{1}{u^{3 / 2} f(u)},
\end{aligned}
$$

with $f(u)=1-\left(u_{\mathrm{KK}} / u\right)^{3}$. Here $T_{8}$ is the D8 $(\overline{\mathrm{D} 8})$ brane tension and we have introduced the dimensionless coordinate $u=R^{3} U /\left(2 \pi \alpha^{\prime}\right)^{2}$. The dimensionless dynamical variables are the real scalar field $\mathcal{T}(u)$ which really becomes "tachyon" in some regime in the bulk and the scalar $h(u)(=$ $2 \pi \alpha^{\prime} l(u) / R^{3}$ with D8 and $\overline{\mathrm{D} 8}$ brane separation $\left.l(u)\right)$ which is related with the transverse coordinate to the D8 $(\overline{\mathrm{D} 8})$ branes describing the "shape" of these branes. We have assumed these are functions of $u$. An important ingredient in (12) is so-called tachyon potential $V(\mathcal{T})$. There are several proposals for this and there is no rigorous derivation to fix its form so far. We here choose one of them:

$$
V(\mathcal{T})=\operatorname{sech}(\sqrt{\pi} \mathcal{T})
$$

One of the important properties equipped for $V(\mathcal{T})$ is that the true vacuum is at $\mathcal{T} \rightarrow \infty$. The effective potential might be obtained from the action (12) by setting $\mathcal{T}^{\prime}(u)=h^{\prime}(u)=0$,

$$
V_{\mathrm{eff}}(\mathcal{T}, h) \sim V(\mathcal{T}) \sqrt{1+u^{3 / 2} f(u) h^{2} \mathcal{T}^{2}}
$$

For large $u$, there is a perturbatively stable minimum at $\mathcal{T}=0$. However, as $u$ decreases, this becomes unstable, since the small- $\mathcal{T}$ expansion in (14) shows the tachyonic behavior like (11). We expect that around that point this tachyonic mode would condense and go to the true vacuum in the potential (13). We could observe this by looking into the detail of the solution of the equation of motion.

\footnotetext{
${ }^{6}$ Strictly speaking, the formulated action is only for the brane-antibrane which are separated along a noncompact direction i.e. $f(U)=1$ in (7a). If the joining point of the branes is far from the $U_{\mathrm{KK}}$, the applicable condition is approximately satisfied.
} 


\subsection{Asymptotic solutions and chiral symmetry breaking}

The equations of motion for $\mathcal{T}(u)$ and $h(u)$ are given as

$$
\begin{aligned}
& 0=\left(\frac{u^{13 / 4} \mathcal{T}^{\prime}(u)}{\sqrt{D[\mathcal{T}(u), h(u)]}}\right)^{\prime}-\frac{u^{13 / 4}}{\sqrt{D[\mathcal{T}(u), h(u)]}}\left\{\frac{1}{V(\mathcal{T})} \frac{\delta V(\mathcal{T})}{\delta \mathcal{T}}\left(D[\mathcal{T}(u), h(u)]-\mathcal{T}^{\prime 2}(u)\right)+h^{2}(u) \mathcal{T}(u)\right\} \\
& 0=\left(\frac{u^{19 / 4} f(u) h^{\prime}(u)}{\sqrt{D[\mathcal{T}(u), h(u)]}}\right)^{\prime}+\frac{u^{13 / 4}}{\sqrt{D[\mathcal{T}(u), h(u)]}}\left\{u^{3 / 2} f(u) \frac{1}{V(\mathcal{T})} \frac{\delta V(\mathcal{T})}{\delta \mathcal{T}} \mathcal{T}^{\prime}(u) h^{\prime}(u)-4 \mathcal{T}^{2}(u) h(u)\right\} .
\end{aligned}
$$

One can find the following asymptotic solutions in the IR regime:

$$
\mathcal{T}(u)=\frac{\sqrt{\pi} u_{0}^{3 / 2} f\left(u_{0}\right)}{4}\left(u-u_{0}\right)^{-2}, \quad h(u)=\sqrt{\frac{26}{\pi u_{0}^{5 / 2} f\left(u_{0}\right)}}\left(u-u_{0}\right)^{1 / 2} .
$$

At the point $u=u_{0}$, D8 and $\overline{\mathrm{D} 8}$ branes are smoothly connected and the filed $\mathcal{T}$ diverges. This implies that the tachyon condensation makes the chiral symmetry breaking [24].

In order to compute the gauge theory quantities, we should look at the behavior of the solutions at large $u$. Since in this regime, $\mathcal{T}$ is massive, we can treat this as a small perturbation from $\mathcal{T}=0$. Then we could obtain the following asymptotic solutions with the integration constants $\left(\mathcal{T}_{+}, \mathcal{T}_{-}\right)$and $\left(h_{0}, h_{1}\right)$ :

$$
\mathcal{T}(u)=\frac{1}{u^{2}}\left(\mathcal{T}_{+} \mathrm{e}^{-h_{0} u}+\mathcal{T}_{-} \mathrm{e}^{h_{0} u}\right), \quad h(u)=h_{0}-h_{1} u^{-9 / 2}
$$

where $h_{0}$ is an asymptotic separation of D8 and $\overline{\mathrm{D}} 8$ branes. From the solution of $\mathcal{T}(u)$, it is obvious that we have to introduce the cut-off for large $u$ for a proper treatment [24].

\subsection{Quark mass, chiral condensate and pion mass}

As we saw in the previous section, in the AdS/CFT correspondence, two independent modes appear in the solution i.e. the non-normalizable mode interpreted as the source term and the normalizable mode which becomes a VEV of the corresponding operator. Here in the solution of $\mathcal{T}(u)$, we could identify $\mathcal{T}_{-}$and $\mathcal{T}_{+}$as the quark mass $m_{\mathrm{q}}$ and the chiral condensate $\left\langle\bar{q}_{\mathrm{L}} q_{\mathrm{R}}\right\rangle$, respectively. By using (6), we can specify the relations:

$$
m_{\mathrm{q}}=\mu\left|\mathcal{T}_{-}\right|, \quad\left\langle\bar{q}_{\mathrm{L}} q_{\mathrm{R}}\right\rangle=\left(\frac{T_{8} V_{4}\left(2 \pi \alpha^{\prime}\right)^{7}}{g_{\mathrm{s}} R^{9}}\right) \frac{4 h_{0}}{\mu} \mathcal{T}_{+},
$$

where we have introduced a mass scale $\mu$.

Let us consider the meson spectrum. These are given by the fluctuation of the $\mathcal{T}(u), h(u)$ and the gauge fields on the flavor branes from the background. In the present case, the background contains nontrivial bi-fundamental scalar field "tachyon". We here consider the lowest pseudo-scalar meson pion. Solving the eigenvalue equations for the corresponding fluctuations, we can estimate the mass of pion in the presence of the quark mass $m_{\mathrm{q}}$. It can be shown further that the pion mass follows the Gell-Mann-Oakes-Renner relation:

$$
M_{\pi}^{2} \propto \frac{m_{\mathrm{q}}\left\langle\bar{q}_{\mathrm{L}} q_{\mathrm{R}}\right\rangle}{f_{\pi}^{2}} .
$$

\section{Desperately searching for scalars}

In a recent work [27], it was found that when a dilaton was implemented into non-linear sigma model - which is valid for low-energy hadron dynamics - to account for the broken scale invariance in 
QCD, the dilaton scalar does two remarkable things: It provides the necessary attraction that binds nucleons in nuclei at low density and tames the strong repulsive core at high density, leading to possible chiral/deconfinement phase transition from hadrons to quark-gluons. This suggests that if holographic approaches to baryonic matter - nucleus, nuclear matter, dense matter etc. - were to make progress, it would be absolutely necessary to have a degree of freedom in bulk theory that represents the scalar figuring in the gauge theory sector.

In the Sakai-Sugimoto model (in fact in all holographic models that we are aware of), such a scalar is missing. The model has the short-distance repulsion known to be present in nature but no mechanism to tame it at high density as in [27]. There have been attempts to bring such scalars into the model, all without success as far as we know. For instance, in [28], the authors obtain in the SakaiSugimoto model an attraction by coupling the gauge fields to a scalar field describing fluctuations of the geometry of the flavor branes. But the effective scalar is more massive than the vector in the theory so that it gives a structure opposite to what's given in [27] at odds with nature. Other attempts [29] to account for correct relevant degrees of freedom in holographic approaches led to properties of hadrons in dense medium that disagree with nature and/or with QCD-motivated models. The culprit here must also be the missing scalar. Finding such a scalar is the most urgent task in applying string theory to dense matter.

\section{Acknowledgment}

We would like to thank the organizers of HNP2011 for the stimulating environment during the conference. YK, TT and DY acknowledge the Max Planck Society (MPG), the Korea Ministry of Education, Science and Technology (MEST), Gyeongsangbuk-Do and Pohang City for the support of the Independent Junior Research Group at the Asia Pacific Center for Theoretical Physics (APCTP). The work of MR is partially supported by the WCU project of the Korean Ministry of Educational Science and Technology (R33-2008-000-10087-0).

\section{References}

1. K. Rajagopal and F. Wilczek, [arXiv:hep-ph/0011333].

2. P. de Forcrand, PoS LAT2009 (2009) 010, [arXiv: 1005.0539 [hep-lat]].

3. E. Shuryak, Prog. Part. Nucl. Phys. 53 (2004) 273, [arXiv:hep-ph/0312227]; Nucl. Phys. A750 (2005) 64, [arXiv: hep-ph/0405066].

4. P. Kovtun, D.T. Son and A.O. Starinets, Phys. Rev. Lett. 94 (2005) 111601, [arXiv:hep-th/0405231].

5. G. 't Hooft, [arXiv: gr-qc/9310026].

L. Susskind, J. Math. Phys. 36 (1995) 6377, [arXiv: hep-th/9409089].

6. G. 't Hooft, Nucl. Phys. B72 (1974) 461.

7. J.M. Maldacena, Adv. Theor. Math. Phys. 2 (1998) 231, [arXiv: hep-th/9711200].

8. J. Polchinski, Phys. Rev. Lett. 75 (1995) 4724, [arXiv: hep-th/9510017].

9. E. Witten, Nucl. Phys. B460 (1996) 335, [arXiv: hep-th/9510135].

10. G.W. Gibbons and K.-I. Maeda, Nucl. Phys. B298 (1988) 741.

D. Garfinkle, G.T. Horowitz and A. Strominger, Phys. Rev. D43 (1991) 3140,

G.T. Horowitz and A. Strominger, Nucl. Phys. B360 (1991) 197.

11. R.G. Leigh, Mod. Phys. Lett. A4 (1989) 2767.

12. L. Susskind and E. Witten, [arXiv:hep-th/9805114].

A.W. Peet and J. Polchinski, Phys. Rev. D59 (1999) 065011, [arXiv: hep-th/9809022].

13. V. Balasubramanian and P. Kraus, Phys. Rev. Lett. 83 (1999) 3605, [arXiv: hep-th/9903190]. J. de Boer, E.P. Verlinde and H.L. Verlinde, JHEP 0008 (2000) 003, [arXiv : hep-th/9912012].

14. S.S. Gubser, I.R. Klebanov and A.M. Polyakov, Phys. Lett. B428 (1998) 105,

[arXiv:hep-th/9802109].

E. Witten, Adv. Theor. Math. Phys. 2 (1998) 253, [arXiv: hep-th/9802150]. 
15. V. Balasubramanian, P. Kraus and A.E. Lawrence, Phys. Rev. D59 (1999) 046003, [arXiv:hep-th/9805171].

16. V. Balasubramanian and P. Kraus, Commun. Math. Phys. 208 (1999) 413, [arXiv: hep-th/9902121].

S. de Haro, S.N. Solodukhin and K. Skenderis, Commun. Math. Phys. 217 (2001) 595, [arXiv:hep-th/0002230].

M. Bianchi, D.Z. Freedman and K. Skenderis, Nucl. Phys. B631 (2002) 159, [arXiv: hep-th/0112119].

17. T. Sakai and S. Sugimoto, Prog. Theor. Phys. 113 (2005) 843, [arXiv: hep-th/0412141]; Prog. Theor. Phys. 114 (2006) 1083, [arXiv: hep-th/0507073].

18. E. Witten, Adv. Theor. Math. Phys. 2 (1998) 505, [arXiv: hep-th/9803131].

19. N. Itzhaki, J.M. Maldacena, J. Sonnenschein and S. Yankielowicz, Phys. Rev. D58 (1998) 046004, [arXiv:hep-th/9802042].

20. D.T. Son and A.O. Starinets, JHEP 0209 (2002) 042, [arXiv: hep-th/0205051].

C.P. Herzog and D.T. Son, JHEP 0303 (2003) 046, [arXiv: hep-th/0212072].

21. O. Aharony, J. Sonnenschein and S. Yankielowicz, Annals. Phys. 322 (2007) 1420, [arXiv: hep-th/0612169].

22. A. Karch and E. Katz, JHEP 0206 (2002) 043, [arXiv: hep-th/0205236].

M. Kruczenski, D. Mateos, R.C. Myers and D.J. Winters, JHEP 0307 (2003) 049, [arXiv: hep-th/0304032]; JHEP 0405 (2004) 041, [arXiv: hep-th/0311270].

J. Babington, J. Erdmenger, N.J. Evans, Z. Guralnik and I. Kirsch, Phys. Rev. D69 (2004) 066007, [arXiv: hep-th/0306018].

23. N. Horigome and Y. Tanii, JHEP 0701 (2007) 072, [arXiv: hep-th/0608198].

D. Yamada, JHEP 0810 (2008) 020, [arXiv:0707.0101[hep-th]].

O. Bergman, G. Lifschytz and M. Lippert, JHEP 0711 (2007) 056,

[arXiv:0708.0326[hep-th]].

M. Rozali, H.H. Shieh, M. Van Raamsdonk and J. Wu, JHEP 0801 (2008) 053, [arXiv:0708.1322 [hep-th]].

K.-Y. Kim, S.-J. Sin and I. Zahed, JHEP 0801 (2008) 002, [arXiv:0708 . 1469 [hep-th] ].

24. R. Casero, E. Kiritsis and A. Paredes, Nucl. Phys. B787 (2007) 98, [arXiv: hep-th/0702155]. O. Bergman, S. Seki and J. Sonnenschein, JHEP 0712 (2007) 037, [arXiv:0708.2839[hep-th]].

A. Dhar and P. Nag, JHEP 0801 (2008) 055, [arXiv:0708.3233[hep-th]]; Phys. Rev. D78 (2008) 066021, [arXiv:0804 .4807 [hep-th]].

25. K. Hashimoto, T. Hirayama and A. Miwa, JHEP 0706 (2007) 020, [arXiv: hep-th/0703024].

O. Aharony and D. Kutasov, Phys. Rev. D78 (2008) 026005, [arXiv: 0803 . 3547 [hep-th]].

K. Hashimoto, T. Hirayama, F.-L. Lin and H.-U. Yee, JHEP 0807 (2008) 089, [arXiv:0803.4192[hep-th]].

26. A. Sen, Phys.Rev. D68 (2003) 066008, [arXiv:hep-th/0303057]. M.R. Garousi, JHEP 0501 (2005) 029, [arXiv: hep-th/0411222].

27. C. Sasaki, H.K. Lee, W.G. Paeng and M. Rho, [arXiv: 1103.0184 [hep-ph]].

28. V.S. Kaplunovsky and J. Sonnenschein, JHEP 1105 (2011) 058, [arXiv: 1003. 2621 [hep-th]].

29. K. Jo, M. Rho, Y. Seo and S.-J. Sin, [arXiv: 1104 .2362 [hep-ph]]. 